\title{
3-D Analytical Calculation of the Torque Between Perpendicular Magnetized Magnets in Magnetic Suspensions
}

\author{
J. L. G. Janssen, J. J. H. Paulides, and E. A. Lomonova \\ Department of Electrical Engineering, Group of Electromechanics and Power Electronics, Eindhoven University of Technology, \\ Eindhoven $5600 \mathrm{MB}$, The Netherlands
}

\begin{abstract}
This paper presents novel equations that enable the direct analytical calculation of the interaction torque between perpendicularly magnetized cuboidal permanent magnets in free space. These expressions complement the expressions for the interaction force and stiffness that are already available in literature and are computationally inexpensive due to their analytical nature. They are suitable in the design and analysis of ironless structures such as certain types of magnetic bearings or for obtaining the peeling torque on the magnets in devices such as voice coil actuators or electron beam focusing devices.
\end{abstract}

Index Terms-Electromagnetic modeling, magnetic forces, magnetic levitation, permanent magnets, torque.

\section{INTRODUCTION}

$\mathbf{M}$ ANY applications require an accurate calculation of the interaction between permanent magnets. Preferably, such calculation should be fast-solving in order to model complex structures or implement advanced optimization techniques. Examples are magnetic suspensions [1], [2], magnet biased microactuators [3], [4], magnetic couplings [5], [6], etc.

Simplified 2-D analytical models meant for radial bearings and couplings were initially proposed by Yonnet [6], [7]. Although these 2-D analytical equations are uncomplicated and require little computational effort, their accuracy remains insufficient in many applications. The development of fully analytical 3 -D models to obtain the interaction force between two parallel magnetized permanent magnets [8], [9] was a significant step forward in terms of accuracy, and due to its analytical properties, it also exhibits low computational costs. Analytical expressions for the circumferential and axial force component of magnetic couplings were proposed in [10].

Force calculations for perpendicular magnetized magnets were proposed in [11] and [12] using the Lorentz force and Virtual work method, respectively. Because the analytical surface charge model is linear, it allows for superposition that enables the user to model the force between multiple magnets with any magnetization direction. Fig. 1 schematically shows this with $30^{\circ}$ rotated magnetization vectors that are expressed as two components along the $x$ - and $z$-axis.

Since these force expressions only produce valid results as long as the magnet edges are not aligned, [13] proposed complementary force expressions for parallel and perpendicular magnetized permanent magnets for such specific cases. Furthermore, [13] proposed analytical expressions for the $3 \times 3$ stiffness matrix $\mathbf{K}$, which is necessary for magnetic suspensions to analyze the performance. The 3-D surface charge model was used by various authors [1], [2], [14] to maximize the force density between permanent magnets, which is useful in the optimization of magnetic suspensions.

Manuscript received February 21, 2011; accepted May 02, 2011. Date of current version September 23, 2011. Corresponding author: J. L. G. Janssen (e-mail: j.l.g.janssen@tue.nl).

Color versions of one or more of the figures in this paper are available online at http://ieeexplore.ieee.org.

Digital Object Identifier 10.1109/TMAG.2011.2154315

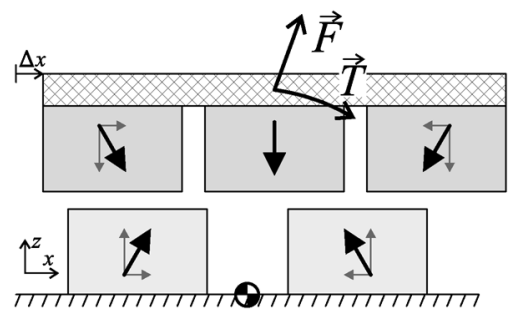

Fig. 1. Schematic 2-D drawing of a magnetic suspension with $30^{\circ}$ rotated magnetization vectors and horizontal displacement $\Delta x$. The force and torque vectors $\vec{F}$ and $\vec{T}$ are also shown.

Especially for magnetic suspensions, an accurate torque computation is eminent. Fig. 1 schematically shows an example of a magnetic suspension with horizontal displacement $\Delta x$. This displacement causes a torque $\vec{T}$ that must be canceled by any external actuators. An accurate prediction of this torque therefore helps to minimize it in the design process. To compute the torque on a permanent magnet as a result of the interaction with another magnet, it is not sufficient to simply multiply the analytically calculated force with an arm because the torque does not act on a single point of the magnet. This was recognized and addressed in two different publications that proposed analytical torque expressions for magnets with parallel magnetization. Reference [15] used Virtual work to obtain analytical expressions for the torque around one of the permanent magnet centers. On the other hand, in [16], the Lorentz force was used to obtain expressions that describe the torque around any given point, and additionally, the necessary complementary expressions for the special case of aligned edges were derived. This paper completes these torque computations with the computation of torque between perpendicular magnetized permanent magnets for any given relative magnet position and torque reference.

This paper is organized as follows. Section II sets up the variables used to model the interaction torque, for which the expressions are subsequently derived in Section III. The discontinuities in these expressions are then discussed and solved in Section IV, followed by the validation in Section V and the conclusions in Section VI.

\section{MODEL DESCRIPTION}

Two cuboidal permanent magnets named PM1 and PM2, respectively, are studied in a Cartesian coordinate system. It is assumed that the edges of these permanent magnets are along 


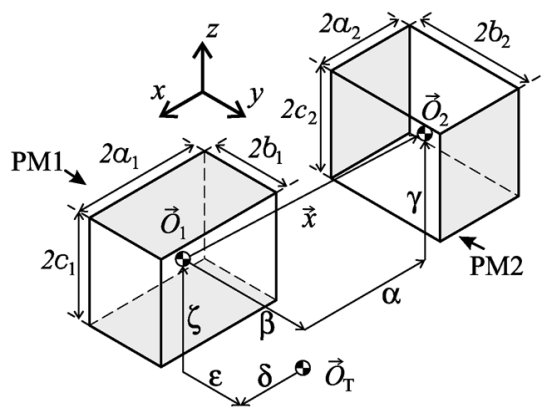

Fig. 2. Definition of the variables used in the torque expressions. Both magnets are magnetized along the $z$-axis.

one of the Cartesian axes and that their respective magnetization vectors $\vec{M}_{1}$ and $\vec{M}_{2}$ are homogeneous, constant, and directed along the $z$ - and $y$-axis, respectively. The dimensions of the permanent magnet are given by $2 a_{1}, 2 b_{1}, 2 c_{1}$ and $2 a_{2}, 2 b_{2}, 2 c_{2}$, respectively (Fig. 2). The center of PM1, $\vec{O}_{1}$, is located at $[0,0,0]^{T}$, and that of PM2, $\vec{O}_{2}$, at $[\alpha, \beta, \gamma]^{T}$ represented by the vector $\vec{x}$. The reference point for the torque calculation, $\vec{O}_{T}$, is located at $[\delta, \epsilon, \zeta]^{T}$. In the charge model [17], each PM is replaced by an equivalent magnetic charge density distribution, $\sigma_{m}$, which is defined as

$$
\sigma_{m}(\vec{x})=\vec{M}_{m} \cdot \overrightarrow{\hat{n}}(\vec{x})=\mu_{0}^{-1} \vec{B}_{\mathrm{r}} \cdot \overrightarrow{\hat{n}}(\vec{x})
$$

where $\overrightarrow{\hat{n}}$ is the normal vector of the PM surfaces, $\vec{B}_{\mathrm{r}}$ is its remanent flux density, and $\mu_{0}$ is the permeability of vacuum. Its main modeling assumptions are that the relative permeability $\mu_{\mathrm{r}}$ equals unity everywhere, and that the magnetization is homogeneous and confined to the permanent magnet materials. Hence, no saturation occurs and the model becomes linear, which allows to use the superposition principle to model complex structures with many magnets.

The vector $\vec{x}$ is the observation point, given by $[x, y, z]^{T}$. In this paper, the torque on PM2 is derived for the case that PM1 is magnetized along the $z$-axis and PM2 is magnetized along the $y$-axis. This completes the torque expressions that were given for parallel magnetization in [16]. For other perpendicular magnetization directions, a rotation of the coordinate system suffices to obtain the torque. Due to the linearity of the models, the superposition principle can easily be implemented to obtain the torque between permanent magnets that are not magnetized exactly along one of the edges.

\section{Analytical TorQue Calculation}

This paper utilizes the Lorentz force method to obtain the analytical torque expressions and derives these expressions for direct torque calculation around any given reference point. The torque calculation is obtained for PM2. A similar approach provides the torque on PM1, which yields an equal but opposite torque result, and is left for the reader. The first step in finding the torque expressions is to obtain the Lorentz force on PM2 that has the form [11]

$$
\begin{aligned}
& \vec{F}(\vec{x})=\iint_{S} \vec{f}\left(\overrightarrow{x^{\prime}}\right) \mathrm{d} S \\
& \vec{f}\left(\overrightarrow{x^{\prime}}\right)=\sigma_{2}\left(\overrightarrow{x^{\prime}}\right) \vec{B}_{1}\left(\overrightarrow{x^{\prime}}\right) .
\end{aligned}
$$

The variable $S$ is the surface of PM2, and $\vec{f}\left(\overrightarrow{x^{\prime}}\right)$ is a virtual force distribution over this surface. The external magnetic flux density that is induced by PM1, $\vec{B}_{1}$, is analytically described in, e.g., [8] and [17] with the charge model.

To obtain correct analytical expressions for the torque on PM2, it necessary to integrate a torque distribution over its surface. This yields the expression

$$
\begin{aligned}
& \vec{T}(\vec{x})=\iint_{S} \vec{t}\left(\overrightarrow{x^{\prime}}\right) \mathrm{d} S \\
& \vec{t}\left(\overrightarrow{x^{\prime}}\right)=\vec{r}\left(\overrightarrow{x^{\prime}}\right) \times \vec{f}\left(\overrightarrow{x^{\prime}}\right) .
\end{aligned}
$$

The arm is given by $\vec{r}\left(\overrightarrow{x^{\prime}}\right)=\overrightarrow{x^{\prime}}-\vec{O}_{T}$ and (4) becomes

$$
\vec{T}(\vec{x})=\iint_{S}\left(\begin{array}{l}
\sigma_{2}\left(\overrightarrow{x^{\prime}}\right)\left(r_{y} B_{z}\left(\overrightarrow{x^{\prime}}\right)-r_{z} B_{y}\left(\overrightarrow{x^{\prime}}\right)\right) \\
\sigma_{2}\left(\overrightarrow{x^{\prime}}\right)\left(r_{z} B_{x}\left(\overrightarrow{x^{\prime}}\right)-r_{x} B_{z}\left(\overrightarrow{x^{\prime}}\right)\right) \\
\sigma_{2}\left(\overrightarrow{x^{\prime}}\right)\left(r_{x} B_{y}\left(\overrightarrow{x^{\prime}}\right)-r_{y} B_{x}\left(\overrightarrow{x^{\prime}}\right)\right)
\end{array}\right) \mathrm{d} S .
$$

After symbolically solving (6), the resulting torque expressions for PM2 obtain the form

$$
\begin{aligned}
\vec{T}(\vec{x})= & \frac{B_{\mathrm{r}_{1}}^{z} B_{\mathrm{r}_{2}}^{y}}{4 \pi \mu_{0}} \\
& \cdot \sum_{i=0}^{1} \sum_{j=0}^{1} \sum_{k=0}^{1} \sum_{l=0}^{1} \sum_{m=0}^{1} \sum_{n=0}^{1}(-1)^{i+j+k+l+m+n} \vec{\xi} .
\end{aligned}
$$

In this equation $B_{\mathrm{r}_{1}}^{z}$ and $B_{\mathrm{r}_{2}}^{y}$ are the remanent flux density components along the $z$ - and $y$-axis of PM1 and PM2, respectively. The vector $\vec{\xi}$ contains the integrand and is separated in three cartesian components $\xi_{x}, \xi_{y}$, and $\xi_{z}$. These integrands are given in (8)-(10) and are substituted in (7)

$$
\begin{aligned}
& \xi_{x}=\frac{1}{12}\left(2 r u^{2}+3 u\left(v^{2}+w^{2}\right)-12 u v\left(C_{v}+v\right) \operatorname{coth}^{-1}\left(\frac{u}{r}\right)\right. \\
& +2 r\left(v^{2}+3 C_{v} v-2 w^{2}\right)-6 u\left(v^{2}+w\left(2 C_{w}+w\right)\right) \\
& \times \log (r-u)-3 C_{w}\left(u^{2}-4 u w+v^{2}+w(w+2 r)\right) \\
& -2 u^{3}-6\left(C_{v}+v\right)\left(u^{2}-w^{2}\right) \operatorname{coth}^{-1}\left(\frac{v}{r}\right) \\
& -12\left(C_{w} v^{2} \operatorname{coth}^{-1}\left(\frac{w}{r}\right)\right. \\
& +u\left(C_{w} v \tan ^{-1}\left(\frac{w}{v}\right)-\left(C_{v}+v\right) w \tan ^{-1}\left(\frac{u v}{w r}\right)\right. \\
& \left.\left.+C_{w} v \tan ^{-1}\left(\frac{u w}{v r}\right)\right)\right) \\
& \left.+6 C_{w}\left(u^{2}+v^{2}\right) \log (w+r)\right) \\
& \xi_{y}=\frac{1}{72}\left(3 v ^ { 3 } \left(2 \log \left(v^{2}+w^{2}\right)+2 \log (u+r)-3\right.\right. \\
& \left.-2 \log \left(v^{2}+w^{2}+2 u(u+r)\right)\right) \\
& +36 v^{2}\left(u-2 C_{w} \tan ^{-1}\left(\frac{u}{v}\right)+C_{w} \tan ^{-1}\left(\frac{u w}{v r}\right)\right) \\
& +36 u v\left(2 C_{u}+u\right) \operatorname{coth}^{-1}\left(\frac{u}{r}\right)-36 C_{u} r v \\
& -9 v\left(8 C_{w} u \log (w+r)+w^{2}\right. \\
& \left.+2 w\left(4 C_{w}+w\right) \log (u+r)+u^{2}-8 C_{w} u\right) \\
& -30 u r v+36\left(u^{2}\left(C_{u}+u\right)-C_{u} w^{2}\right) \operatorname{coth}^{-1}\left(\frac{v}{r}\right) \\
& +2\left(12 w^{2}\left(3 C_{w}+w\right) \tan ^{-1}\left(\frac{u}{w}\right)\right. \\
& +18 C_{w}\left(u^{2}+w^{2}\right) \tan ^{-1}\left(\frac{w}{u}\right)
\end{aligned}
$$




$$
\begin{aligned}
&+6 w\left(w^{2}+3 C_{w} w-3 u\left(2 C_{u}+u\right)\right) \tan ^{-1}\left(\frac{u v}{w r}\right) \\
&+u\left(3 u^{2} \log \left(u^{2}+w^{2}\right)\right. \\
&-3 u^{2} \log \left(u^{2}+w^{2}+2 v(v+r)\right) \\
&+18 u C_{w} \tan ^{-1}\left(\frac{v w}{u r}\right)-5 u^{2} \\
&-3 w\left(18 C_{w}+7 w\right) \\
&\left.\left.\left.+6\left(u^{2}+3 w\left(2 C_{w}+w\right)\right) \log (r-v)\right)\right)\right) \\
& \xi_{z}=\frac{1}{36}\left(6 w\left(v^{2}-3 u\left(2 C_{u}+u\right)\right)+18 u w\left(2 C_{u}+u\right)\right. \\
& \times \log (r-u)+w^{3}-3 w\left(3 v^{2}+w^{2}\right) \log (u+r) \\
&+3\left(6 C_{u}+u\right) r w-6 v\left(v^{2}-3 u\left(2 C_{u}+u\right)\right) \\
& \times \tan ^{-1}\left(\frac{w}{v}\right)+6 v\left(v^{2}+3 u\left(2 C_{u}+u\right)\right) \\
& \times \tan ^{-1}\left(\frac{u w}{v r}\right)-6\left(2 u^{3}+3 C_{u}\left(u^{2}-v^{2}\right)\right) \\
& \times \log (w+r)-18\left(C_{v}+v\right) \\
& \times\left(\left(\tan ^{-1}\left(\frac{w}{u}\right)+\tan ^{-1}\left(\frac{v w}{u r}\right)\right) u^{2}+2 v u\right. \\
& \quad-2 u v \log (w+r)-2 v^{2} \tan ^{-1}\left(\frac{u}{v}\right) \\
&+v^{2} \tan ^{-1}\left(\frac{u w}{v r}\right)-3 w u \\
&+w(-2 v \log (u+r)+2 u \log (r-v) \\
&+w\left(2 \tan ^{-1}\left(\frac{u}{w}\right)+\tan ^{-1}\left(\frac{w}{u}\right)\right. \\
&\left.\left.\left.\left.\quad+\tan ^{-1}\left(\frac{u v}{w r}\right)\right)\right)\right)\right) .
\end{aligned}
$$

The variables $\vec{C}, u, v, w$, and $s$ are given by

$$
\begin{aligned}
\vec{C} & =\left(\begin{array}{l}
C_{u} \\
C_{v} \\
C_{w}
\end{array}\right)=\left(\begin{array}{c}
(-1)^{i} a_{1}-\delta \\
(-1)^{k} b_{1}-\epsilon \\
(-1)^{m} c_{1}-\zeta
\end{array}\right) \\
u & =\alpha-(-1)^{i} a_{1}+(-1)^{j} a_{2} \\
v & =\beta-(-1)^{k} b_{1}+(-1)^{l} b_{2} \\
w & =\gamma-(-1)^{m} c_{1}+(-1)^{n} c_{2} \\
r & =\sqrt{u^{2}+v^{2}+w^{2}}
\end{aligned}
$$

with the variables defined in Fig. 2. Although these equations seem rather complicated, they enable an extremely fast and accurate calculation of the torque on a PM in the presence of a magnetic field of another PM.

\section{DisContinUities IN THE ANALYTICAL EXPRESSIONS}

Equations (12)-(15) show that certain combinations of $i \ldots n, a_{1} \ldots c_{2}$, and $\alpha \ldots \gamma$ cancel each other and $u, v$, or $w$ become zero, i.e., when the edges of two PMs are aligned. Fig. 3 shows such a situation in which (12) and (14) reduce to

$$
\begin{array}{r}
\left.u\right|_{i=j, a_{1}=a_{2}, \alpha=0}=0 \\
\left.w\right|_{m=n, c_{1}=c_{2}, \gamma=0}=0
\end{array}
$$

and the expressions (8)-(10) become undefined. A fully analytical, hence computationally inexpensive, equation in these situations is the most accurate and fast solution to this problem and

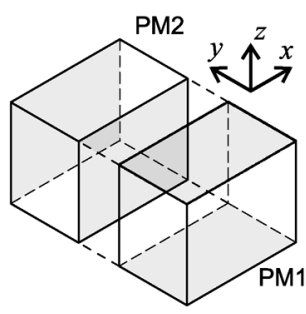

Fig. 3. Alignment of the PM edges causes problems in the standard force, stiffness, and torque expressions, hence complementary terms for these situations are necessary.

has been derived for the force, stiffness, and torque of parallel magnetized magnets in [13] and [16].

The analytical equations for $\vec{\xi}$ in the special cases that $u=$ $v=0, u=w=0$, or $v=w=0$ are found by solving for the limits of (8)-(10). The resulting equations are valid equations in these points

$$
\begin{aligned}
\lim _{\substack{u \rightarrow 0 \\
v \rightarrow 0}} \vec{\xi}_{x}=\frac{1}{24}\left(3 C_{v} w^{2} \log \left(w^{2}\right)\right. \\
-2\left(3 C_{v} w^{2} \log \left(-\sqrt{w^{2}}\right)\right. \\
\left.\left.+3 C_{w} w\left(w+2 \sqrt{w^{2}}\right)+4\left(w^{2}\right)^{3 / 2}\right)\right)
\end{aligned}
$$

$\lim _{\substack{u \rightarrow 0 \\ v \rightarrow 0}} \vec{\xi}_{y}=-\frac{1}{8} w\left(C_{u} w \log \left(w^{2}\right)\right.$

$$
\left.-2\left(\pi \sqrt{w^{2}} C_{w}+C_{u} w \log \left(-\sqrt{w^{2}}\right)\right)\right)
$$

$\lim _{\substack{u \rightarrow 0 \\ v \rightarrow 0}} \vec{\xi}_{z}=\frac{1}{72}\left(2 w\left(w^{2}+9\left(2 C_{u}-C_{v} \pi\right) \sqrt{w^{2}}\right)\right.$

$$
\left.-3 w^{3} \log \left(w^{2}\right)\right)
$$

$\lim _{\substack{u \rightarrow 0 \\ w \rightarrow 0}} \vec{\xi}_{x}=\frac{1}{12} v\left(-3 C_{w} v+6 C_{w} v \log \left(-\sqrt{v^{2}}\right)\right.$

$$
\begin{gathered}
\left.+2 \sqrt{v^{2}}\left(3 C_{v}+v\right)\right) \\
\lim _{\substack{u \rightarrow 0 \\
w \rightarrow 0}} \vec{\xi}_{y}=\frac{1}{24}\left(v^{3} \log \left(v^{2}\right)-3\left(v^{3}+4 C_{u} \sqrt{v^{2}} v\right)\right) \\
\lim _{\substack{u \rightarrow 0 \\
w \rightarrow 0}} \vec{\xi}_{z}=\frac{1}{4} C_{u} v^{2} \log \left(v^{2}\right)
\end{gathered}
$$$$
\lim _{\substack{v \rightarrow 0 \\ w \rightarrow 0}} \vec{\xi}_{x}=\frac{1}{24}\left(-4 u^{3}-6 C_{w} u^{2}-3 u^{2}\left(C_{v}-2 C_{w}\right) \log \left(u^{2}\right)\right.
$$$$
\left.+6 C_{v} \log \left(-\sqrt{u^{2}}\right) u^{2}+4\left(u^{2}\right)^{3 / 2}\right)
$$

$\lim _{\substack{v \rightarrow 0 \\ w \rightarrow 0}} \vec{\xi}_{y}=\frac{1}{72} u^{2}\left(3\left(3 C_{u}+5 u\right) \log \left(u^{2}\right)\right.$

$$
\left.-2\left(5 u+9\left(C_{u}+u\right) \log \left(-\sqrt{u^{2}}\right)\right)\right)
$$

$\lim _{\substack{v \rightarrow 0 \\ w \rightarrow 0}} \vec{\xi}_{z}=-\frac{1}{12} u^{2}\left(3 C_{u}+2 u\right) \log \left(u^{2}\right)$.

It is observed that the logarithmic terms with $\log \left(-\sqrt{x^{2}}\right)$ produce imaginary terms. However, the symmetry in (7) cancels these imaginary term, and, as such, only real torque results are obtained. With these equations, a fully analytical and continuous torque calculation method for cuboidal permanent magnets is obtained. In combination with the analytical continuous force and stiffness equations from [13] and the parallel torque 
TABLE I

DimENSIONS OF THE MEASURED MAGNETS, DEFINED IN Fig. 2

\begin{tabular}{|c|c|c|c|c|c|}
\hline $2 a_{1}$ & $10 \mathrm{~mm}$ & $2 a_{2}$ & $14 \mathrm{~mm}$ & $\alpha$ & $0 \mathrm{~mm}$ \\
\hline $2 b_{1}$ & $26 \mathrm{~mm}$ & $2 b_{2}$ & $26 \mathrm{~mm}$ & $\beta$ & $-8 \mathrm{~mm}$ \\
\hline $2 c_{1}$ & $14 \mathrm{~mm}$ & $2 c_{2}$ & $10 \mathrm{~mm}$ & $\gamma$ & $15 \mathrm{~mm}$ \\
\hline$\vec{M}_{2}$ & $M \vec{e}_{z}$ & $\vec{M}_{2}$ & $M \vec{e}_{x}$ & $B_{r_{1}}=B_{r_{2}}$ & $1.23 \mathrm{~T}$ \\
\hline
\end{tabular}

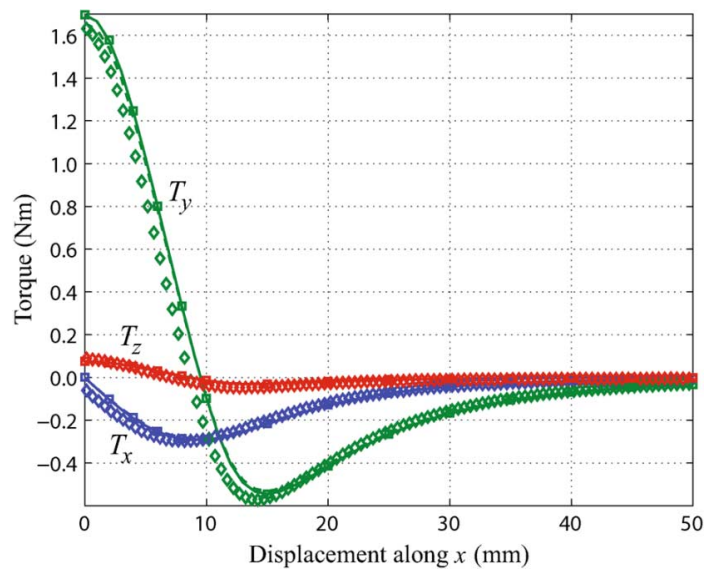

Fig. 4. Torque results of the FEM model ( $\square$ ), Maxwell Stress results $(\ldots)$, the analytical charge model proposed in this paper $(-)$, and measurements $(\diamond)$.

computations in [16], a versatile analytical tool for the analysis, design, and optimization of PM bearings with cuboidal magnets has been developed.

\section{EXPERIMENTAL VALIDATION}

Practical validation measurements are the key to successful modeling techniques. For this reason, the equations in this paper are not only compared to results from numerical Maxwell Stress integration on the analytical field (mesh of $0.2 \mathrm{~mm}$ ) and finite element (FE) results, but also with experimental measurements that were described in [11]. This is performed for two magnets with the dimensions shown in Table I, with the reference point for the torque defined by the vector $[0,0,-47]^{T} \mathrm{~mm}$. This point is the center point of the load cell that has been used, which is the ATI-Mini40-SI40-2 force and torque transducer. This sensor holds one of the magnets in a certain $x$ - and $y$-position, while the other magnet is moved along the $x$-direction by a linear drive. Fig. 4 shows the high correspondence between all methods, hence, verifies the fast-solving torque calculation proposed in this paper. A very small difference of some percents is caused by measuring errors and the relative permeability of the permanent magnets, which equals 1.03 rather than 1 as the model assumes. The solving time of the analytical model per position step was $2 \mathrm{~ms}$, that with Maxwell Stress $40 \mathrm{~ms}$, and the FE results took $167 \mathrm{~s}$ per step.

\section{CONCLUSION}

This paper presents novel analytical torque equations for cuboidal permanent magnets with perpendicular magnetization. It includes additional equations for the nonsolving terms occurring in the special cases in which the standard equations are undefined. Together, they enable the direct calculation of the torque on the permanent magnets with respect to any torque reference point.
The analytical results are verified with numerical (FEM), seminumerical models (Maxwell Stress) and with experimental measurements, which illustrate the accuracy of these fast-solving analytical equations. Although they seem rather complex at first sight, the obtained equations solve extremely fast. This makes them very suitable for the design and optimization of permanent magnet devices such as planar static magnetic bearings and vibration isolators, especially when combined with the force, stiffness, and torque expressions derived in previous publications.

\section{ACKNOWLEDGMENT}

This work was supported by IOP-EMVT 06225 by AgentschapNL, which is part of the Dutch Ministry of Economical Affairs.

\section{REFERENCES}

[1] J. L. G. Janssen, J. J. H. Paulides, and E. A. Lomonova, "Passive magnetic suspension limitations for gravity compensation," J. Syst. Design Dynam., vol. 3, no. 4, pp. 671-680, 2009.

[2] W. Robertson, B. S. Cazzolato, and A. C. Zander, "Maximizing the force between two cuboid magnets," IEEE Magn. Lett., vol. 1, p. $9000102,2010$.

[3] C. Dieppedale, B. Desloges, H. Rostaing, J. Delamare, O. Cugat, and J. Meunier-Carus, "Magnetic bistable micro-actuator with integrated permanent magnets," in Proc. IEEE Sensors, Oct. 2004, vol. 1, pp. 493-496.

[4] S. Shetye, J. Agashe, and D. Arnold, "Investigation of microscale magnetic forces for magnet array self-assembly," IEEE Trans. Magn., vol. 43, no. 6, pp. 2713-2715, Jun. 2007.

[5] P. Elies and G. Lemarquand, "Analytical optimization of the torque of a permanent-magnet coaxial synchronous coupling," IEEE Trans. Magn., vol. 34, no. 4, pp. 2267-2273, Jul. 1998.

[6] J.-P. Yonnet, "Permanent magnet bearings and couplings," IEEE Trans. Magn., vol. 17, no. 1, pp. 1169-1173, Jan. 1981.

[7] J.-P. Yonnet, S. Hemmerlin, E. Rulliere, and G. Lemarquand, "Analytical calculation of permanent magnet couplings," IEEE Trans. Magn., vol. 29, no. 6, pp. 2932-2934, Nov. 1993.

[8] G. Akound and J. P. Yonnet, "3D analytical calculation of the forces exerted between two cuboidal magnets," IEEE Trans. Magn., vol. 20, no. 5, pp. 1962-1964, Sep. 1984.

[9] F. Bancel, "Magnetic nodes," J. Appl. Phys., vol. 32, pp. 2155-2161, Jun. 1999.

[10] P. Elies and G. Lemarquand, "Comparison of the torque of flat airgap synchronous couplings versus the magnetization direction of the magnets," IEEE Trans. Magn., vol. 35, no. 1, pp. 548-556, Jan. 1999.

[11] J. L. G. Janssen, J. J. H. Paulides, and E. Lomonova, "Analytical calculation of interaction force between orthogonally magnetized permanent magnets," Sensor Lett., vol. 7, no. 3, pp. 442-445, Aug. 2009.

[12] H. Allag and J. P. Yonnet, "3D analytical calculation of interactions between perpendicularly magnetized magnets: Application to any magnetization direction," Sensor Lett., vol. 7, no. 4, pp. 486-491, Aug. 2009.

[13] J. L. G. Janssen, J. J. H. Paulides, and E. A. Lomonova, "Analytical force and stiffness calculations for magnetic bearings and vibration isolation," in Computer Field Models of Electromagnetic Devices, ser. Studies in Applied Electromagnetics, E. Napieralska-Juszczak and S. Wiak, Eds., 1st ed. Amsterdam, The Netherlands: IOS Press, 2010, pp. 502-511.

[14] J.S. Agashe and D. P. Arnold, "A study of scaling and geometry effects on the forces between cuboidal and cylindrical magnets using analytical force solutions," J. Phys. D, Appl. Phys., vol. 41, no. 105001, pp. 1-9, 2008.

[15] H. Allag and J.-P. Yonnet, "3-d analytical calculation of the torque and force exerted between two cuboidal magnets," IEEE Trans. Magn., vol. 45, no. 10, pp. 3969-3972, Oct. 2009.

[16] J. L. G. Janssen, J. J. H. Paulides, J. C. Compter, and E. A. Lomonova, "3D analytical calculation of the torque between permanent magnets in magnetic bearings," IEEE Trans. Magn., vol. 46, no. 6, pp. 1748-1751, Jun. 2010.

[17] E. P. Furlani, Permanent Magnet and Electromechanical Devices: Materials, Analysis and Applications, 6th ed. London, U.K.: Academic, 2001. 